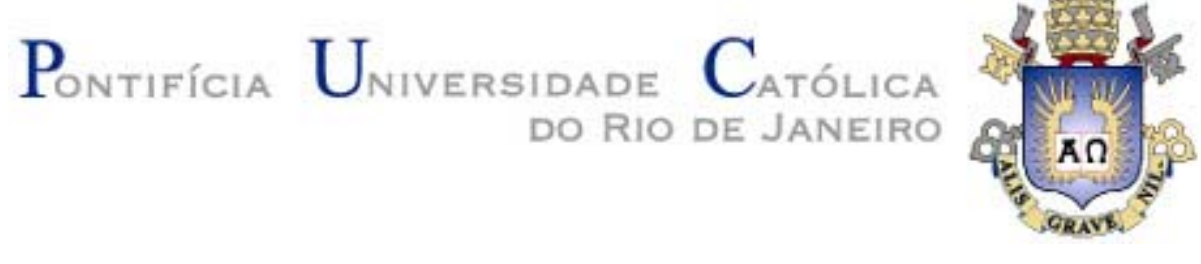

Dennis Seiji Taquemori

ISO 9001: Eficiência ou Legitimidade?

Dissertação de Mestrado

Dissertação apresentada ao Programa de Pósgraduação em Administração de Empresas da PUC-Rio como requisito parcial para obtenção do título de Mestre em Administração de Empresas.

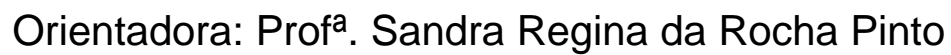




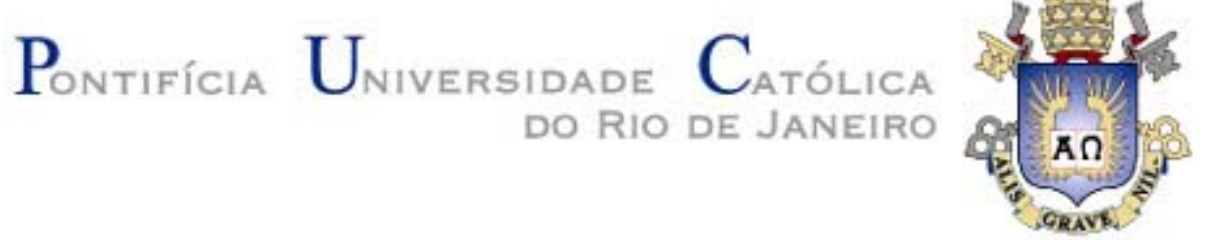

Dennis Seiji Taquemori

\section{ISO 9001: Eficiência ou Legitimidade?}

Dissertação apresentada como requisito parcial para obtenção do grau de Mestre pelo Programa de Pós-Graduação em Administração de Empresas da PUC-Rio. Aprovada pela Comissão Examinadora abaixo assinada

Prof ${ }^{a}$. Sandra Regina da Rocha Pinto

Orientadora

Departamento de Administração - PUC-Rio

Prof. José Roberto Gomes da Silva Departamento de Administração - PUC-Rio

Marcelo Milano Falcão Vieira FVG-Rio

Prof. Nizar Messari Vice-Decano de Pós-Graduação do CCS

Rio de Janeiro, 17 de março de 2009. 
Todos os direitos reservados. É proibida a reprodução total ou parcial do trabalho sem autorização da universidade, do autor e da orientadora.

\section{Dennis Seiji Taquemori}

Ficha Catalográfica

Taquemori, Dennis Seiji

ISSO 9001 : eficiência ou legitimidade ? / Dennis Seiji Taquemori ; orientadora: Sandra Regina da Rocha Pinto. - 2009.

101 f. : il. (color.) ; $30 \mathrm{~cm}$

Dissertação (Mestrado em Administração)Pontifícia Universidade Católica do Rio de Janeiro, Rio de Janeiro, 2009.

Inclui bibliografia

1. Administração - Teses. 2. ISSO 9000. 3. Teoria institucional. 4. Legitimidade. 5. Isomorfismo. 6. Desinstitucionalização. 7. Eficiência. I. Pinto, Sandra Regina da Rocha. II. Pontifícia Universidade Católica do Rio de Janeiro. Departamento de Administração. III. Título.

CDD: 658 
Para meus pais pela educação e formação que me proporcionaram.

Para Laura pela compreensão e apoio. 


\section{Agradecimentos}

À Universidade Pontífice Católica (PUC-Rio) e à minha orientadora Professora Sandra da Rocha-Pinto, sem os quais esse trabalho não teria se concretizado. 


\section{Resumo}

Taquemori, Dennis Seiji; Pinto, Sandra Regina da Rocha. ISO 9001: Eficiência ou Legitimidade? Rio de Janeiro, 2009. 101p. Dissertação de Mestrado - Departamento de Administração, Pontifícia Universidade Católica do Rio de Janeiro.

Um volume cada vez maior de práticas institucionais tem surgido com vistas a regular ou melhorar a atuação das organizações ou reduzir os custos de transação. Contudo, se observa que essas práticas, não necessariamente resultam maior eficiência. Com o intuito de evitar uma análise reducionista e mecanicista, optou-se por utilizar como base do estudo a teoria neo-institucional, por levar em consideração os seguintes aspectos: legitimidade; eficiência; isomorfismo; desinstitucionalização. Nesse contexto, a organização ISO representa um interessante objeto de estudo por ser internacionalmente legitimada e transformar diversas crenças e valores em regras e normas que se tornam mitos e cerimônias. Além disso, a pesquisa procurou, principalmente, analisar dois elementos aparentemente contrários na teoria institucional: eficiência e legitimidade. Para tal, utilizou-se como referência a percepção de especialistas quanto à eficiência da implementação da norma internacional para sistemas de gestão da qualidade, ISO 9001. Essa ISO, ao mesmo tempo em que parece legitimar as organizações que se certificam, é tida como um modelo de gestão que se propõe a gerar maior eficiência. Como conclusão, o dilema eficiência versus legitimidade na prática da ISO 9001 é analisado e são apresentados possíveis indícios de um processo de desinstitucionalização: práticas inovadoras; normas mais abrangentes; e, alteração de valores institucionais. Dessa forma, esse estudo representa uma contribuição tanto à academia por aplicar a teoria institucional à prática da ISO 9001 quanto para as organizações, por apresentar possíveis melhorias na implementação dessa norma e a tendência de sua provável substituição.

\section{Palavras-chave}

ISO 9000; teoria institucional; legitimidade; isomorfismo; desinstitucionalização; eficiência. 


\section{Abstract}

Taquemori, Dennis Seiji; Pinto, Sandra Regina da Rocha (Advisor). ISO 9001: Efficiency or Legitimacy? Rio de Janeiro, 2009. 101p. MSc. Dissertation - Departamento de Administração, Pontifícia Universidade Católica do Rio de Janeiro.

An increasing number of institutional practices has emerged to rule or improve organizational performances and reduce transaction-costs. However, these practices don't necessarily enhance efficiency. The neo-institutional theory is chosen as the groundwork for this study in order to avoid a reductionist, mechanical analysis, and also for taking into account the following aspects: legitimacy; efficiency; isomorphism; and deinstitutionalization. The ISO organization represents an interesting subject to study since it is legitimated worldwide, and transforms several values and beliefs into organizational rules and norms that become myths and ceremony. Moreover, this research aimed at analyzing two seemingly opposite elements in the institutional theory: efficiency and legitimacy. In order to accomplish its objectives, the study applied as reference the perception of specialists in relation to the efficiency of implementation of the international standards for quality management systems known as ISO 9001. This ISO seems to legitimate organizations that become certified and, at the same time, it's believed to be a management model intended to produce more efficiency. As an outcome of the study, the dilemma efficiency versus legitimacy in the practice of ISO 9001 is analyzed, and then possible evidences of a process of deinstitutionalization are presented: innovative practices; more extensive standards; and modification of institutional values. Therefore this study represents a contribution to academic matters through applying the institutional theory to the practice of ISO 9001, in as much as to organizations, since it presents possible improvements in the implementation of this standard, and the tendency of its possible substitution.

\section{Keywords}

ISO 9001; institutional theory; legitimacy; isomorphism; deinstitutionalization; efficiency. 


\section{Sumário}

1 Introdução 12

1.1. A motivação e o problema da pesquisa 12

1.2. Objetivos 14

1.3. Relevância do estudo 15

1.4. Delimitações do estudo 16

$\begin{array}{ll}\text { 1.5. Estrutura da dissertação } & 18\end{array}$

2 Referencial teórico 19

2.1. A teoria institucional 19

2.1.1. O institucionalismo 20

2.1.2. Legitimidade 26

2.1.3. Isomorfismo 30

2.1.4. O velho e o novo institucionalismo 36

2.1.5. Desinstitucionalização 40

$\begin{array}{ll}\text { 2.1.6. Teoria e prática } & 47\end{array}$

3 Metodologia $\quad 49$

3.1. Linha epistemológica e estratégia de pesquisa 49

3.2. Limitações e contornos 50

3.3. Universo e sujeitos 51

3.4. Coleta de dados 54

3.5. Tratamento de dados $\quad 56$

4 Análise e discussão dos resultados $\quad 57$

4.1. A institucionalização da organização 58

4.2. A institucionalização da ISO 9001

4.3. A legitimidade da ISO 9001

4.4. A eficiência da ISO $9001 \quad 65$

4.5. O processo de isomorfismo pela ISO 9001

4.6. Indícios do processo de desinstitucionalização 79 
5 Considerações finais 91

5.1. Conclusão 92

5.2. Sugestão para pesquisas futuras 94

6 Referências bibliográficas 95

6.1. Sites pesquisados 99

$\begin{array}{ll}7 \text { Anexo } & 100\end{array}$ 


\section{Lista de figuras}

Figura 1 - Quantidade de padrões publicados 13

Figura 2 - Total de certificados ISO 9001 no mundo 17

Figura 3 - Total de certificado ISO 9001 na América Latina 17

Figura 4 - As origens e elaboração das estruturas organizacionais formais 23

Figura 5 - Sobrevivência organizacional 28

Figura 6 - Etapas de formação de um campo organizacional 31

Figura 7 - Os efeitos do isomorfismo institucional nas organizações $\quad 35$

Figura 8 - Pressões para Desinstitucionalização 45

Figura 9 - Teoria e prática $\quad 47$

Figura 10 - Evolução de certificados ISO 9001 emitidos no Brasil 61 


\section{Lista de quadros}

Quadro 1 - Combinando ambiente técnico e institucional 29

Quadro 2 - O Velho e o Novo Institucionalismo 37

Quadro 3 - Soluções e limitações na adoção de

práticas institucionalizadas $\quad 40$

Quadro 4 - Respostas estratégicas aos processos institucionais 41

Quadro 5 - Antecedentes Institucionais e Respostas

Estratégicas Previstas 43

Quadro 6 - Antecedentes da Desinstitucionalização 46

Quadro 7 - Perfil demográfico e educacional dos entrevistados $\quad 52$

Quadro 8 - Perfil profissional dos entrevistados 53

Quadro 9 - Roteiro semi-estruturado da pesquisa $\quad 55$

Quadro 10 - Respostas estratégicas à exigência da ISO 9001

Quadro 11 - Fatores limitantes à adoção da ISO 9001

Quadro 12 - Pressões para a desinstitucionalização da ISO $9001 \quad 88$ 